\title{
EL AULA DE FORMACIÓN PERMANENTE EN ARQUEOLOGÍA DE LA UCO: INVESTIGACIÓN, FORMACIÓN Y DIFUSIÓN PARA TODOS LOS PÚBLICOS $^{1}$
}

\author{
Dra. Ana Ruiz Osuna y Belén Vázquez Navajas²
}

\section{Resumen:}

El objetivo del presente texto es mostrar las bases y los principios que rigen el Aula de Formación Permanente en Arqueología de la Facultad de Filosofía y Letras de Córdoba; una alternativa pedagógica que nace con el firme propósito de ofrecer -a todos los niveles educativos y edades- propuestas didácticas que faciliten la comprensión de la Historia, el acercamiento a la ciencia arqueológica y la creación de recursos divulgativos de calidad abiertos al conjunto de la sociedad.

Palabras clave:

Arqueología, Aula de Formación, Investigación, Difusión, Educación

\section{Abstract:}

The aim of this paper is to show the ruling principles of the Permanent Archaeological Training Room of the Faculty of Arts of Cordoba. It is an educational alternative which intends to offer to all ages and levels of education instructional materials to facilitate the understanding of History, the approach to Archaeology and the development of high quality dissemination tools for all society.

\section{Key words:}

Archaeology, Training Room, Research, Dissemination, Education

\section{Objetivos iniciales}

En la actualidad, la sociedad se encuentra inmersa en un proceso de transformaciones profundas, del que debemos salir reinventados y fortalecidos. En medio de esta catarsis, la educación se ha convertido en uno de los grandes temas de debate; los modelos de enseñanza de nuestro país están en el punto de mira de Europa y de la ciudadanía española, quien, consciente de su valor

1 Este trabajo se inscribe en el marco del Proyecto de Investigación Del registro estratigráfico a la sociedad del conocimiento: el patrimonio arqueológico urbano y rural como agente de desarrollo sostenible, financiado por la Dirección General de Investigación Científica y Técnica y Subdirección General de Proyectos de Investigación. Ministerio de Economía y Competitividad. Gobierno de España, en su convocatoria de 2013 (Ref.: HAR2013-43389-R; Subprograma Retos de la Sociedad).

2 Área de Arqueología. Universidad de Córdoba 
e importancia, demanda, cada vez más, la creación de nuevas estrategias pedagógicas, accesibles a todas las esferas sociales.

Las tendencias más recientes, con apoyo especial de las TIC's, están introduciendo mejoras en los métodos de aprendizaje, permitiendo a los sujetos enfrentarse a la búsqueda de respuestas de forma autónoma. Sin embargo, al mismo tiempo, las nuevas tecnologías están implantando entre las generaciones más jóvenes un enciclopedismo feroz, un hecho que hay que combatir con propuestas educativas novedosas y creativas, pero sin olvidar, en ningún caso, los valores de la lectura, la reflexión y el pensamiento crítico. De este modo, se podrá luchar contra los vicios heredados de la educación tradicional tales como la pasividad o la superficialidad ${ }^{3}$.

Junto a los colegios, institutos y escuelas superiores, la Universidad debe igualmente implicarse de pleno en este proceso y en la construcción de un marco educativo que posibilite la formación de individuos competentes dentro de un contexto social, económico y cultural en constante cambio. Es por ello que entre los fines prioritarios de la docencia universitaria debe destacar la superación de los modelos clásicos, encorsetados en compartimentaciones y saberes demasiado artificiales. Sin duda, la Enseñanza Superior tiene que actuar como factor de progreso y modernización, afianzando su compromiso con la sociedad que la genera y sostiene y, sobre todo, abriendo sus puertas con actividades destinadas a distintos públicos.

Por este motivo, y con el deseo de lograr aprendizajes significativos, el Grupo de Investigación Sísifo (PAIDI HUM-236) del Área de Arqueología de la Universidad de Córdoba, dirigido por el Catedrático Desiderio Vaquerizo Gil, trabaja en una alternativa pedagógica para la enseñanza de esta disciplina histórica, que ha tomado cuerpo recientemente gracias a la creación de un Aula de Formación Permanente en Arqueología en la Facultad de Filosofía y Letras; un auténtico laboratorio de investigación, formación y difusión de la ciencia arqueológica, basado en el trabajo conjunto entre docentes y discentes, y diseñado para hacer penetrar los conceptos teóricos tanto mediante el contacto directo con las fuentes escritas y materiales, como a través de las dinámicas metodológicas propias de esta rama humanística.

La importancia de convertir la Arqueología en un recurso útil, accesible y rentable; la conveniencia de procurar al alumnado nuevas formas de implicación epistemológica y práctica; la localización y las particularidades de nuestro centro de trabajo, en pleno corazón del casco histórico de la capital cordobesa;

3 En este sentido, y siguiendo la estela de países pioneros como Finlandia (GRIPENBERG y LIZARTE, 2012), en algunas escuelas españolas han comenzado a implantarse ya modelos educativos alejados de los clásicos exámenes y de las clases magistrales. Destacan en concreto tres colegios catalanes de la Orden Jesuita que recientemente han adoptado nuevos programas pedagógicos basados en la flexibilidad, los tutores multidisciplinares, el "descubrimiento guiado" y el trabajo en equipo. 
y la posibilidad de ofrecer a diferentes sectores las herramientas y los recursos creados en el marco del Grupo de Investigación Sísifo, justifican de todo punto la puesta en marcha de un Aula de Formación como la que aquí se presenta, cuyos objetivos de partida son:

Consolidar un laboratorio constante de propuestas docentes, que permita, con ayuda de las nuevas tecnologías, mejorar la manera de impartir clases, evaluar correctamente el aprendizaje del alumnado y configurar materiales didácticos interdisciplinares de calidad que inicien a los participantes en el mundo de la investigación arqueológica.

- Transferir el conocimiento arqueológico y cultural generado desde la Universidad a la sociedad, especialmente a los alumnos de todos los niveles de enseñanza, pretendiendo convertir la institución universitaria en un referente de la vida cultural de Córdoba, donde se conjuguen la tradición y la innovación.

- Enriquecer la formación de los alumnos involucrándolos de forma activa en el proceso de enseñanza y aprendizaje, al tiempo que puedan desarrollar habilidades individuales y grupales, como la capacidad crítica, el espíritu emprendedor y la agilidad mental, o valores como la perseverancia, el compañerismo y la tolerancia.

- Crear sinergias con otras instituciones educativas en busca de modelos pedagógicos que abran nuevas perspectivas dentro del campo de la Educación y de la Arqueología, capaces incluso de generar empleo y autofinanciación con la que retroalimentar la investigación.

\section{Porque nadie nace sabiendo...}

El proyecto del Aula de Formación Permanente en Arqueología parte de la amplia experiencia investigadora y docente del Grupo de Investigación Sísifo de la Universidad de Córdoba ${ }^{4}$. Desde hace años, nuestro equipo de profesionales mantiene un completo programa de actividades enfocadas a la difusión y transferencia del conocimiento histórico, cuyos resultados han sido siempre muy positivos. Fruto de esta labor, comenzamos a desarrollar en el año 2011 el Proyecto de Cultura Científica Arqueología Somos Todos que, más allá de reforzar el papel de la Arqueología como ciencia, propone acciones divulgativas enfocadas a recrear la evolución urbana de Córdoba, una de las ciudades más antiguas y paradigmáticas del Mediterráneo. Con este fin, ofrecemos anualmente distintos ciclos de conferencias, talleres infantiles,

4 Vid. www.gruposisifo.com.

5 Vid. www.arqueologíasomostodos.com y el artículo de D. Vaquerizo Gil en este mismo volumen. 
certámenes, exposiciones, rutas temáticas y expediciones arqueológicas, así como un conjunto de recursos didácticos realizados con el máximo rigor científico, pero adaptados a todas las edades.

Además de nuestra propia trayectoria, tuvimos en cuenta el trabajo desarrollado con anterioridad en otros ámbitos académicos, tales como los Laboratorios de Arqueología Experimental de la Universidad de Valladolid, de la Universidad de Barcelona en Vendrell (MORER et alii 1999) y Alorda Park (POU, SANMARTí Y SANTACANA 1995) o los Laboratorios de Arqueología Prehistórica de la Universidad Rey Juan Carlos y la Universidad Jaume I (OLÀRIA I PUYOLES 1994). Pero, sin duda, destaca por su gran trayectoria y alcance el Laboratorio de Arqueología Experimental de la Universidad Autónoma de Madrid; centro pionero en el desarrollo de actividades experimentales en relación al material arqueológico, en el que se llevan a cabo estudios de tecnología experimental y funcionalidad, así como acciones de transferencia mediante la elaboración y uso de instrumentos antiguos. Igualmente interesantes son las actividades derivadas del Laboratorio de Arqueología y Enseñanza (hoy integrado en el Centro de Estudios sobre Patrimonio Arqueológico de la Prehistoria de la Universidad Autónoma de Barcelona), fruto de una iniciativa conjunta del entonces Departamento de Antropología Social y Prehistoria y la Unidad de Didáctica de las Ciencias Sociales, con la voluntad de llevar a cabo una puesta en común de las experiencias y reflexiones de las diferentes partes implicadas en los proyectos divulgativos en torno a la Arqueología y, de forma más genérica, en torno al Patrimonio Histórico o las estrategias que promovió el Laboratorio de Arqueología y Formas Culturales de la Universidad de Santiago de Compostela (CRIADO, BÓVEDA 2000), preocupado por la formación arqueológica en los ciclos de postgrado.

Conocidas y altamente valoradas son también las propuestas pedagógicas con base histórico-arqueológica vinculadas a centros museísticos, yacimientos y parques arqueológicos. Entre las más populares, por su nivel de experimentación y su habilidad para conectar con toda clase de públicos, destacan el Centro de Investigación de Altamira y el Centro de Interpretación del yacimiento de Atapuerta. En el caso de los museos nos parece justo mencionar las labores desempeñadas por el Museo Arqueológico de Tarragona, el Museo Egipcio de Barcelona o los Talleres de Arqueología Experimental Prehistórica del Museo de San Isidro en Madrid (VELÁZQUEZ, CONDE, BAENA 2004.; MARTÍN, CUARTERO 2008), en colaboración con el Laboratorio de Arqueología Experimental de la UAM. Precisamente algunos miembros de este último Laboratorio son los responsables del proyecto Arqueodidat, especializado en la creación de recursos formativos y educativos para entidades museísticas, centros de interpretación y universidades (MARTíN et alii 2012; ROVIRA 2011-2012).

En estos antecedentes era importante contar con la experiencia de otros grupos de investigación en la puesta en marcha de talleres didácticos y arqueódromos, aplicados tanto a universitarios como a alumnos de las enseñanzas obligatorias. 
Uno de los pioneros fue el Taller de Arqueología 4 de la Universitat de València, que durante 1994 y 1996 desarrolló experiencias basadas en la introducción de los métodos y conocimientos propios de la Arqueología en la Enseñanza Secundaria, con el objeto de facilitar el aprendizaje en Historia a través de la recreación de los modos de vida del pasado - pinturas parietales prehistóricas - y, fundamentalmente, del método arqueológico gracias a la excavación de un yacimiento simulado del neolítico (GIL, PÉREZ Y FIÈRREZ 1996). En esta misma línea están el Taller de Verano Arqueología y Arquitectura de Monte Urgul (Donostia-San Sebastián), organizado por el Centro de Estudios Arkeolan y la Escuela Técnica Superior de Arquitectura de la Universidad del País Vasco, como un programa de iniciación a la Arqueología destinado a estudiantes de arquitectura, aunque de interés para cualquier estudiante universitario de otras disciplinas (NOAIN2002-2003), y el Arqueódromo de la Universidad de las Islas Baleares, consistente en un yacimiento simulado para acercar a los escolares de $4^{\circ}$ de ESO y $1^{\circ}$ de Bachillerato a las distintas fases de una excavación real (GARCÍA et alii 2011). En un apartado especial encontramos los Campos de Aprendizaje del Departamento de Educación de la Generalitat de Catalunya, donde se promueven actividades educativas en espacios naturales y culturales emblemáticos del territorio catalán. En el Campo situado en La Noguera (Lérida), por citar uno de los ejemplos más señalados, se reproduce la vida cotidiana de esta zona durante la Prehistoria y la Edad Media, así como las dificultades sufridas durante la Guerra Civil (BARDAVIO, GONZÁLEZ 2008).

Una apuesta mayor, y fuera de nuestra posibilidades, han sido las reconstrucciones de yacimientos completos, una práctica muy extendida por determinadas regiones de Europa, como el Centro de Arqueología Experimental de Lejre (Dinamarca) (MADSEN 1981), el Buster Ancient Farm (Reino Unido) (REYNOLDS 1979; 1988-89) o el famoso Archéodrome de Borgoña (Francia) (DAVID 1998), creado a base de una serie de reproducciones arquitectónicas arquetípicas de distintos momentos históricos donde investigadores llevan a cabo sus trabajos sobre arqueología experimental a la vista y con participación del público asistente; una experiencia que está, sin duda, en la base del proyecto que está dando vida a un castillo medieval en Guédelon (Francia) (MINARD, FOLCHER 2003).

\section{El arqueódromo de la UCO y sus talleres didácticos}

La creación del Aula de Formación Permanente en Arqueología en la Facultad de Filosofía y Letras de Córdoba debe ser entendida como la culminación de una serie de propuestas divulgativas que, desde hace años, llevamos desarrollando dentro y fuera de las dependencias de dicho edificio. Estas acciones se han ido diseñando con el objetivo de introducir en las dinámicas docentes métodos de 
actuación que faciliten la comprensión de conceptos de carácter históricoarqueológico de forma rápida, amena y activa.

Por una parte, una materia con tan alto contenido instrumental como la Arqueología tenía que permitir al alumnado estar en contacto con el medio físico, ya fuera con un yacimiento real o a través de recreaciones; de este planteamiento surgió nuestro primer proyecto: la construcción de un arqueódromo ${ }^{6}$ durante el primer cuatrimestre del año académico 2012-2013, levantado gracias a la concesión de un Proyecto de Innovación Docente y al patrocinio de la Fundación Española para la Ciencia y la Tecnología y de la Fundación Obra Social "la Caixa".

La idea de utilizar una excavación simulada para transmitir y trabajar los conocimientos, habilidades y valores relacionados con la Arqueología comenzó a extenderse por ámbitos anglosajones y franceses hace ya algún tiempo, y ha sido repetida en España con posterioridad en varias ocasiones. Nuestro arqueódromo, a diferencia de otros y de los denominados "cajones de arena" ${ }^{\text {", }}$, recrea un yacimiento arqueológico a escala real con varias fases de ocupación. La primera de ellas queda representada por una necrópolis romana en la que se disponen dos enterramientos, uno de cremación y otro de inhumación. A este cementerio se le superpone una vivienda típica andalusí, compuesta por un zaguán; un patio, con andén perimetral y pozo de agua; una salón-alcoba, con suelo y paredes a la almagra; una letrina y una fosa séptica situada en una calle pavimentada. Finalmente, las estructuras islámicas aparecen cortadas en el patio por un pozo contemporáneo construido a base de ladrillo y cemento. Sobre los restos inmuebles, así como en el interior de las tumbas y los pozos, se ubican varios objetos -artefactos y ecofactos- asociados a cada una de las etapas representadas. La excavación se encuentra permanentemente cubierta por varias capas de arena y gravilla, de manera que los participantes puedan reproducir y asimilar la teoría y las técnicas arqueológicas desde el primer paso.

La mayoría de los arqueódromos reconstruyen hábitats domésticos y tumbas prehistóricas de la Edad del Bronce. Si bien nos hemos alejado de dicho periodo histórico a la hora de elegir el motivo principal de nuestro yacimiento, nos hemos

6 En el año 2011 construimos en las dependencias del Real Jardín Botánico de Córdoba un primer yacimiento simulado, el cual nos proporcionó la experiencia y la confianza suficientes para embarcarnos en este segundo arqueódromo.

7 Una versión reducida pero igualmente didáctica de las reconstrucciones arqueológicas son los "cajones de arena", en los que se sitúan pequeñas piezas que deben ser localizadas y/o extraídas. Algunos llegan a adquirir un tamaño considerable y son especialmente útiles en espacios cerrados como museos (Museo Arqueológico de Tarragona; Centro de Interpretación de Los Bañales). Muchos de ellos sirven además como complemento a las visitas de exposiciones temporales (Arqueódromo Cerro del Villar en Málaga) o yacimientos y parques arqueológicos (Cercadilla en Córdoba; Gilena en Sevilla). Nosotros mismos fabricamos hace unos años una de estas cajoneras en el marco del proyecto Arqueología somos todos. 
decantamos igualmente por un contexto residencial, por cuanto entendíamos que sería mucho más fácil de reconocer y entender, en especial por el público infantil. Era también importante introducir una fase de necrópolis que aproximara a nuestros "excavadores" al mundo de la muerte y la escatología, ambas ligadas al hombre desde el origen de la Humanidad.
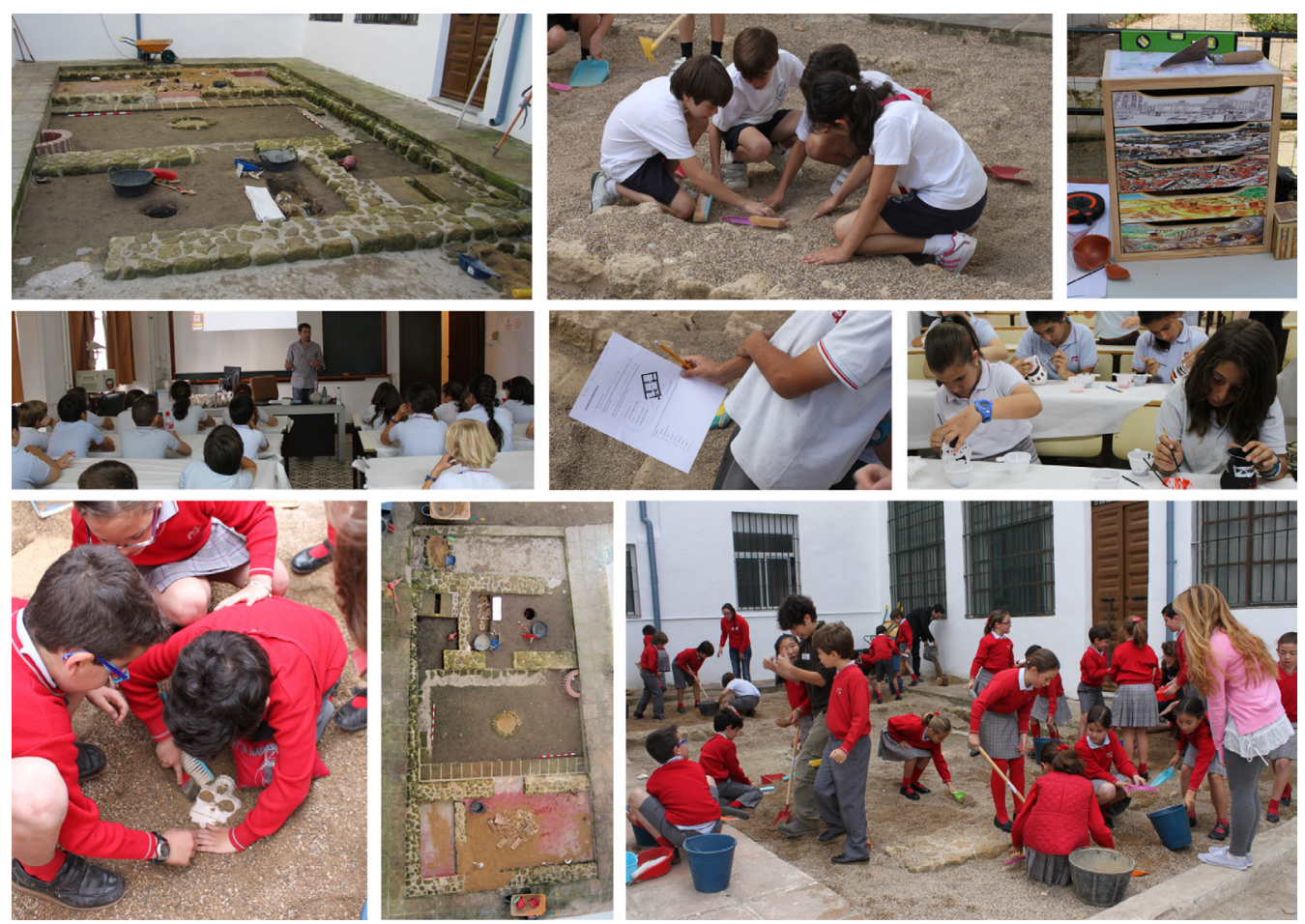

Fig. 1. Experiencias didácticas de escolares en el "arqueódromo".

Una novedad esencial de nuestro arqueódromo es la ausencia de cuadriculación artificial a la hora de organizar y abordar el trabajo arqueológico. Los espacios limitados controlados por tres o cuatro alumnos resultan ideales para comprender el método científico de la excavación. Sin embargo, en nuestro caso particular, hemos optado por centrarnos en la experiencia real que supone participar en una intervención arqueológica urbana en una ciudad histórica como Córdoba. Así se pueden explicar con mayor facilidad la necesidad de tomar las cotas topográficas, la apertura de sondeos, la formación de estratigrafías naturales y artificiales o el porqué de la superposición de estructuras.

Asimismo, hay que tener en cuenta que, frente al uso de un yacimiento real, esta simulación ex novo nos ha liberado de los límites jurídicos y administrativos que imponen, justamente, las leyes patrimoniales y urbanísticas, permitiéndonos, 
por un lado, acoger a "excavadores" noveles en un proceso arqueológico sin poner en peligro el registro material $y$, por otro, recrear una y otra vez una misma excavación. Junto a ello, nuestro arqueódromo permite incluso introducir y jugar con distintas variables que enriquecen considerablemente el discurso metodológico, como la cronología del ajuar de las tumbas, la posición del esqueleto, el cegamiento de vanos, la compartimentación de espacios o la introducción de procesos post-deposicionales.

El arqueódromo de la Facultad de Filosofía y Letras de Córdoba ha sido ya el eje motor de muchas actividades programadas tanto para las prácticas de los alumnos de los grados de Historia, Historia del Arte y Gestión Cultural como para la realización de los talleres didácticos organizados para colegios, niños y familias desde el Proyecto de Cultura Científica Arqueología Somos Todos ${ }^{8}$ (Fig. 1). En el transcurso de la tercera edición de dicho proyecto, financiado de nuevo por la Fundación Española para la Ciencia y la Tecnología, pudimos además reordenar todas estas experiencias y transformarlas en un completo catálogo de talleres ${ }^{9}$, que han quedado englobados en dos bloques temáticos. En primer lugar, ofrecemos varios talleres arqueológicos como complemento directo a las tareas realizadas en el arqueódromo, en los que se continúan remarcando las labores propias del profesional de la Arqueología; entre los más novedosos destacan el Taller de Estratigrafía, el de Epigrafía y el de Dibujo y/o Reconstrucción de Piezas Cerámicas. En segundo lugar, Ilevamos a cabo toda una serie de talleres de corte histórico que inciden en determinados aspectos de la vida diaria de las sociedades del pasado, como el de Pequeños Artesanos, Mosaicos, Candiles y Lucernas, Escritura en el Tiempo, Numismática, Alicatando al-Andalus o Verde y Manganeso.

En nuestro afán por extrapolar modelos, hemos trasladado estos talleres más allá de los muros de la Facultad en múltiples ocasiones (Fig. 2). Dentro de la ciudad hemos acudido a los Centros Cívicos y organizado sesiones de dinamización infantil en el marco de diversos programas, como la Noche de los Investigadores de la Universidad de Córdoba o las I Jornadas de Arqueología en Familia, desarrolladas en la Ciudad de los Niños; este parque infantil, diseñado

8 Los monitores que imparten los talleres son, en todos los casos, licenciados en Historia, Historia del Arte, Humanidades u otros estudios similares, con experiencia en Arqueología y contratados al efecto, que cuentan en todo momento con la ayuda de los miembros del Grupo de Investigación Sísifo, tanto en la fase de preparación y diseño de contenidos como en el desarrollo de los mismos. A ellos se suman los Alumnos Colaboradores y los Voluntarios Culturales de la Facultad de Filosofía y Letras como apoyo logístico.

9 Este dossier presenta de manera clara las ventajas de la Arqueología como ciencia trasversal, la cual se adapta como ninguna a la evaluación por competencias, facilitando la evaluación de contenidos como el análisis e interpretación de distintos tipos de fuentes, el desarrollo del pensamiento lógico y el razonamiento científico, el manejo de vocabulario específico, la búsqueda de explicaciones multicausales, el conocimiento de culturas pasadas o la concienciación del patrimonio. 
para el entretenimiento de los más pequeños, se ha convertido en uno de los emplazamientos más visitados por las familias cordobesas, donde, al margen de dichas jornadas, hemos coordinado otro gran número de acciones.
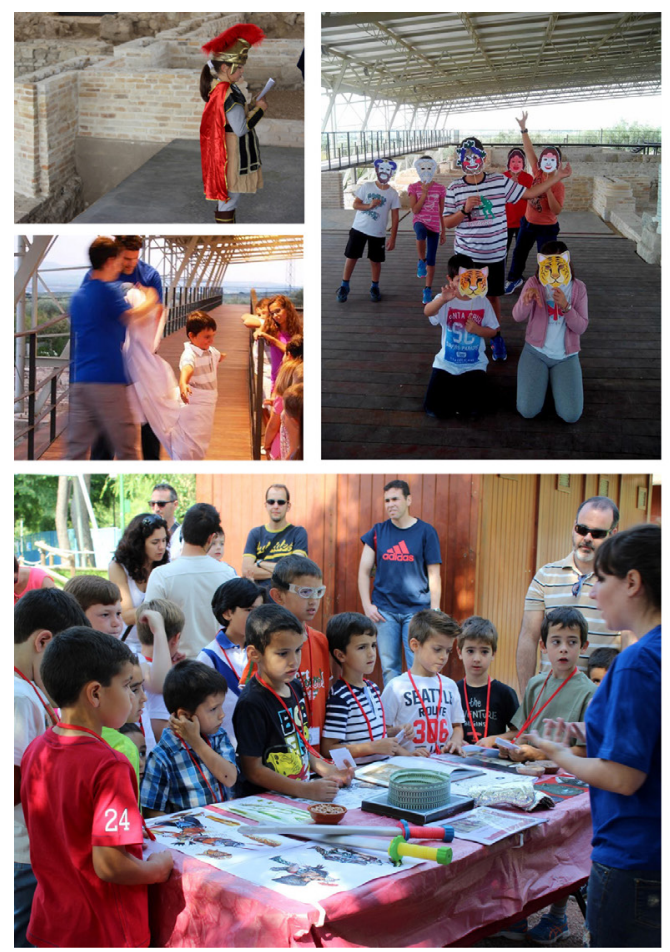
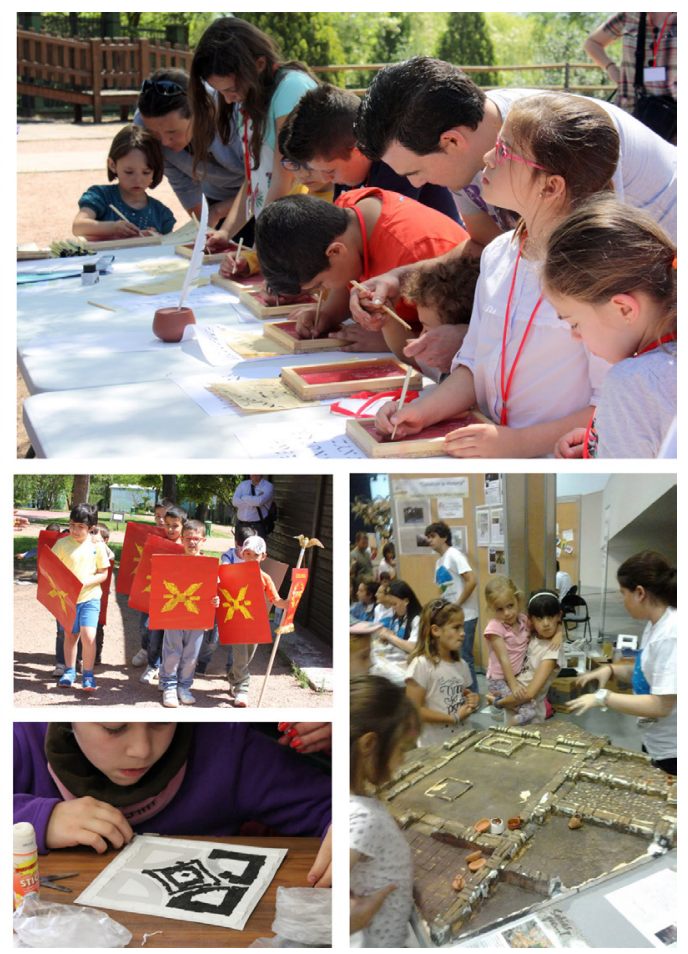

Fig. 2. Algunos de los talleres didácticos desarrollados fuera de nuestra Facultad.

También hemos estado presentes en varias localizaciones de la provincia de Córdoba como Peñarroya-Pueblonuevo, con talleres sobre Arqueología Industrial; Fuenteobejuna, desde donde nos solicitaron completar las visitas escolares a la Feria del Libro y la presentación del Cuaderno Didáctico del Museo Histórico Local; o la Villa Romana de Fuente Álamo (Puente Genil), en la que hemos ejecutado talleres infantiles y actividades nocturnas insertadas en su programación de verano Noches en la Villa y en la celebración del Día Internacional del Turismo. Pero tal vez, uno de nuestros hitos más importantes, por encontrarse fuera de nuestro ámbito habitual de trabajo y por la distinción del lugar, fue la oportunidad de protagonizar una de las Ventanas a la Ciencia del Parque de las Ciencias de Granada, donde, en relación con nuestra exposición Mira por donde pisas: Arqueología somos todos, preparamos un programa didáctico especial para el Día de Andalucía bajo el lema Conoce el patrimonio arqueológico andaluz. 
El éxito de estas iniciativas, de las que se han hecho eco muchas instituciones, colectivos y medios de comunicación autonómicos y nacionales, queda patente en los gráficos de asistencia, y es que, a lo largo de estos años hemos realizado más de 100 talleres atendiendo a casi 3300 participantes (Fig. 3).

Lugar de celebración

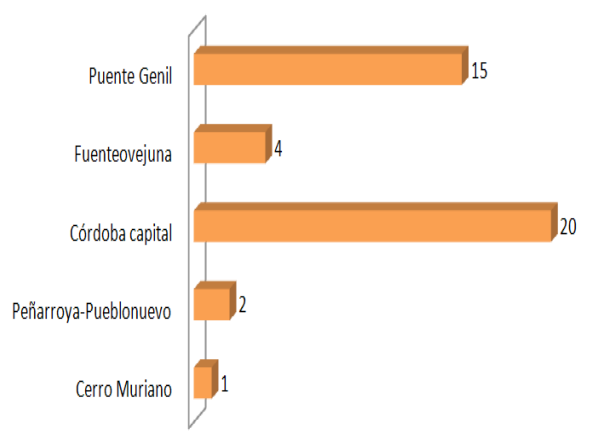

\section{№ de niños}

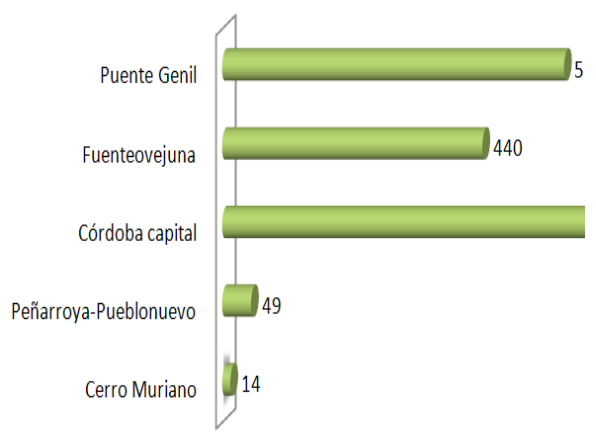

\section{Edades de los participantes}

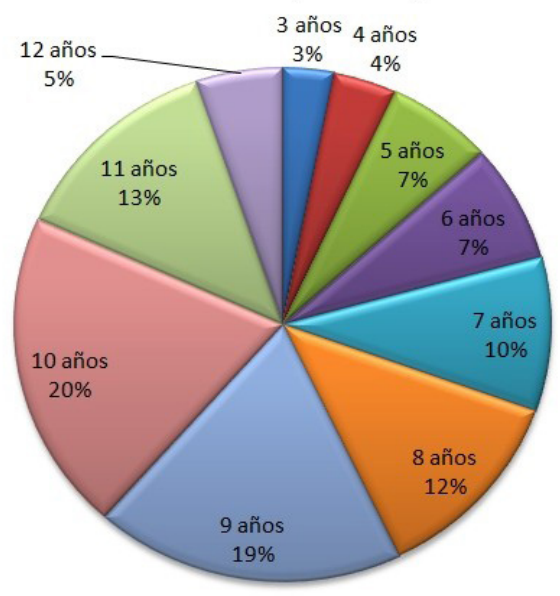

Fig. 3. Gráficos de los talleres infantiles realizados entre 2013 y 2014.

\section{Hacia el Aula de Formación Permanente en Arqueología}

El deseo de ampliar y afianzar nuestros conocimientos, junto con el compromiso de seguir ofreciendo lo mejor de nosotros mismos, hizo que, 
con el apoyo del Decanato de la Facultad de Filosofía y Letras de Córdoba, nos embarcáramos en un nuevo y ambicioso proyecto que contemplaba el arqueódromo y los talleres didácticos como una parte más de un completo plan pedagógico que encuadramos bajo el nombre de Aula de Formación Permanente en Arqueología, cuyas primeras acciones han sido ya puestas en marcha. Esta experiencia piloto, conllevará además en un futuro la cubrición de nuestro yacimiento simulado, ubicado actualmente a la intemperie en uno de los patios de la Facultad, y el acondicionamiento definitivo del Aula I, con la que se encuentra comunicado y en la que venimos realizando nuestros talleres ${ }^{10}$.

La metodología planteada desde el principio para regir esta Aula, sin más dotación presupuestaria que la que podemos derivar de algunos de los proyectos del Grupo de Investigación, ha sido: el fomento de la investigación de campo y gabinete; la formación sólida de profesionales presentes y futuros asociados al mundo de la Arqueología; y la divulgación del Patrimonio HistóricoArqueológico. En este sentido, el trabajo del Aula ha quedado dividido en tres grandes líneas de trabajo:

Investigación. Cualquier labor que tenga como objetivo final la trasmisión y la divulgación de la producción científica debe asentarse en un marco teórico definido y contrastado, por lo que consideramos de vital importancia investigar previamente los mecanismos de difusión más punteros y eficaces, de modo que pudiéramos cumplir a la perfección con los cometidos del Aula; una tarea ardua pero muy fructífera en la que seguiremos siempre inmersos para mejorar y enmendar nuestros fallos y limitaciones.

De igual forma, otra de las metas que nos marcamos fue la transferencia del conocimiento generado por el Grupo de Investigación Sísifo a la comunidad educativa, especialmente a los profesores de Enseñanzas Primarias y Secundarias, para los cuales estamos elaborando contenidos didácticos que podrán obtener en formato de libre acceso a través de nuestras plataformas webs. A ello hay que sumar la creación de una comisión de trabajo integrada por investigadores y profesionales de la Arqueología, pedagogos, ilustradores, maestros y alumnos universitarios con los que articularemos una serie de herramientas ${ }^{11}$ que permitirán al profesorado confeccionar su propio itinerario curricular y planificar visitas, clases y viajes de estudios de calidad.

Formación. Dentro de nuestras prioridades ocupa un lugar preferente el fomento de la educación y la formación, con el convencimiento absoluto de que ambas se hallan en la base del progreso social y económico. Teniendo en cuenta la trasversalidad de la disciplina arqueológica, trabajamos para

10 Este proyecto se encuentra aprobado por Consejo del Centro a la espera de financiación.

11 Ha sido configurada una primera encuesta sobre Patrimonio y Educación Obligatoria que se está haciendo llegar a todos los profesores de Primaria y Secundaria del terrritorio andaluz. 
conseguir que nuestra Aula de Formación llegue a convertirse en una escuela de Arqueología de primer orden, donde se impartan cursos especializados de temáticas dispares (bases de datos, recreaciones virtuales, turismo histórico, técnicas de datación, etc.) y, a su vez, se inculquen valores esenciales como el respeto por el Patrimonio y la diversidad cultural. Gracias a ello, se podrá dotar también a los participantes de instrumentos intelectuales con los que enfrentarse a las distintas situaciones del día a día.

Hasta la fecha, hemos Ilevado a cabo en el Aula las prácticas de algunas asignaturas del Grado de Historia, Historia del Arte o Gestión Cultural, a las que se sumarán en los próximos meses una serie de seminarios. No obstante, consideramos que la formación impartida desde el Aula debe estar abierta a cualquier centro educativo, agrupación, asociación, profesional libre o estudiantes de otras titulaciones de la Universidad de Córdoba o fuera de ella. Por este motivo, contemplamos igualmente organizar en futuras ocasiones talleres sobre la enseñanza de la ciencia arqueológica para educadores ${ }^{12}$; cursos de actualización para guías turísticos e intérpretes del Patrimonio; actividades de asesoramiento para colectivos y empresas; o clases adicionales para los alumnos integrados en programas internacionales como PRESHCO o en la Cátedra Intergeneracional de la Universidad de Córdoba.

Divulgación. Además de atender a la investigación arqueológica de alto rango y a la educación, el Aula de Formación Permanente en Arqueología pretende difundir al conjunto de la sociedad el saber adquirido por los miembros de nuestro Grupo de Investigación. Con esta filosofía hemos comenzado a ofrecer talleres abiertos a toda la ciudadanía, sin distinciones ni limitaciones. Estas actividades están fundamentadas en las realizadas desde del proyecto Arqueología Somos Todos, pero procurando incorporar cada trimestre nuevas propuestas, como es el caso de los talleres arqueogastronómicos de época romana e islámica que hemos iniciado para el público adulto.

La elaboración de material didáctico y divulgativo ha sido otro paso obligado para el buen funcionamiento del Aula. Recientemente hemos editado un "Cuaderno de Trabajo" (Fig. 4) que permite a los participantes en nuestros talleres entender con mayor facilidad las fases del proceso científico arqueológico: desde la formación de un yacimiento hasta su posterior documentación, incluyendo la recogida y tratamiento de los materiales recuperados y el análisis de laboratorio e interpretación de los restos; juegos, curiosidades, dibujos y pequeños textos adaptados a distintos niveles que refuerzan el discurso oral y las actividades prácticas. De igual forma, se están configurando varias Unidades Didácticas sobre las distintas etapas históricas de Córdoba (prerromana, romana,

$12 \mathrm{Al}$ respecto cabe señalar que hemos sido incluidos en un proyecto Erasmus+ liderado por el I.E.S. Fidiana de Córdoba, donde se nos ha pedido impartir un curso de cultura romana para profesores, alumnos y padres, así como actuar como asesores científicos del proyecto All roads lead to Rome. 
tardoantigua, medieval-islámica y bajomedieval-cristiana). Estos programas están siendo diseñados por un equipo multidisciplinar conformado, entre otros, por expertos en educación, que nos estan asesorando y ayudando a seleccionar los objetivos basicos, los contenidos necesarios y las mejores pautas metodológicas para divulgar nuestro Patrimonio.

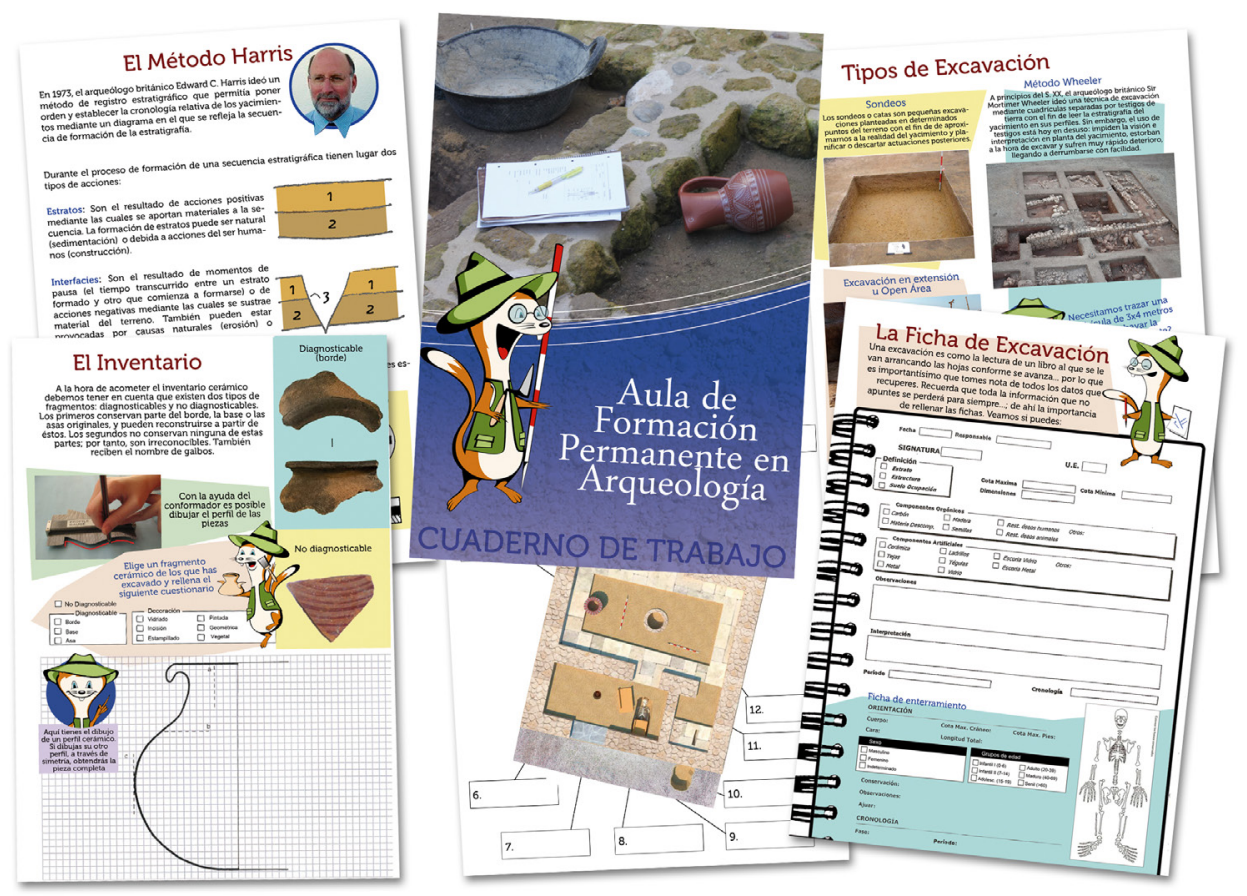

Fig. 4. Algunas capturas del "Cuaderno de Trabajo" del Aula de Formación Permanente en Arqueología de la UCO.

\section{Aprender para difundir, difundir para enseñar: algunas reflexiones finales}

Es una realidad constatada que la tradición pedagógica en España ha infravalorado el papel de la Arqueología en los currículos educativos, donde apenas ha tenido presencia, considerándola sólo como un medio más para acceder a la Historia. Sin embargo, los que nos dedicamos por entero a esta ciencia sabemos que se trata de un método de aprendizaje en sí mismo, basado en la observación, el análisis, la formulación de hipótesis y la verificación de datos, convirtiendo al mundo que nos rodea en una fuente inagotable de información que, bien leída e interpretada, permite extraer conclusiones sobre nuestras formas de ser, habitar y pensar. 
Las propuestas didácticas generadas en el seno de Arqueología Somos Todos han tratado de aprovechar esta versatilidad y presentar la Arqueología de un modo asequible y entretenido. No obstante, es necesario reflexionar ahora sobre ciertas cuestiones inherentes a nuestras propias acciones; por una parte, el peso que deberían ostentar las medidas de divulgación y concienciación patrimonial en el desarrollo de actividades formativas; por otra, la configuración de equipos multidisciplinares que planteen estrategias y herramientas para tal fin; $y$, finalmente, la conveniencia de aumentar en los programas escolares la presencia de la ciencia arqueológica. Estos tres planteamientos nos conducen a una misma y única afirmación: el extraordinario valor de una educación íntegra y plural desde las edades más tempranas, que actúe como nexo de unión entre el Patrimonio y la sociedad, un binomio emergente en el panorama nacional y europeo en el que tienen que involucrarse todo tipo de agentes sociales y culturales, desde profesores e investigadores hasta representantes políticos.

Es bien sabido que sólo se protege y estudia lo que se conoce y se valora, por lo que las administraciones públicas deben incentivar los programas destinados al análisis, protección y divulgación del Patrimonio Histórico-Arqueológico. Pero más allá de ello, no cabe duda de que son los centros educativos los entornos más adecuados para trabajar y fomentar estos conceptos y hacerlo, además, de manera transversal. Son ya muchos los casos los que han demostrado la interesante y necesaria relación entre arqueólogos y maestros, que debería potenciarse también con la implicación en el sistema escolar de museos, yacimientos, parques arqueológicos, escuelas-taller y universidades, lo que redundaría en una mayor valorización de la Arqueología; un tema que ocupó ya intensos debates en países como Inglaterra o Italia hace algunos años (GIL et alii 1996.). En el recorrido que hizo F. López Ambite (2000) por la Educación Secundaria en España se ponía de manifiesto cómo la Arqueología apenas aparecía mencionada en el "concepto $n^{\circ} 2$ del bloque $n^{o} 4$ de las Sociedades Históricas", que abarcaba desde la hominización y el Neolítico hasta la Grecia y la Roma clásicas. Una vacío de contenidos al que hacía igualmente referencia G. Ruiz Zapatero (1995) a través del concepto "pasado excluido".

A este silencio casi total de la Arqueología en los currículos escolares, debemos sumar otro problema cuyo origen se establece en la primera renovación didáctica de los años 80, momento en el que surge una diatriba severa en la enseñanza de esta materia que parte de la diferenciación entre Historia como pasado, entendida como una rama humanística que recrea e investiga los pueblos y hechos del pasado, e Historia como objeto de enseñanza, es decir, una disciplina mediante la cual el alumno asimila la Historia para educarse (LÓPEZ 2000). Esta diferencia ha provocado una cierta incomprensión entre los defensores de la primera opción, más tradicionalistas, y los que buscan un cambio pedagógico, donde no prime tanto el conocimiento exhaustivo de cada una de las etapas históricas, sino que la 
propia Historia sirva de modelo de análisis y aprendizaje trasversal. Precisamente, en esta última línea argumental se fueron centrando las experiencias arqueológicas en el aula en ámbito anglosajón, extendidas al territorio español a partir de los 90 con las visitas puntuales a museos.

Hoy día, tanto la enseñanza como la propia idiosincrasia del Patrimonio han hecho que estas "salidas museísticas" no sean el único método efectivo para la comprensión y asunción de valores y conocimientos históricos. Así, entre las posibles alternativas de dinamización del Patrimonio Cultural se encuentran las actividades realizadas en yacimientos arqueológicos, ya sean urbanos o rurales, donde los escolares pueden tener un contacto directo con los vestigios materiales in situ. Esa relación con el medio juega un papel fundamental en el proceso de enseñanza-aprendizaje, permitiendo a los alumnos iniciarse en la Historia y en la construcción de sus propias conciencias. Para ello, es importante tener en cuenta que, más allá de la consolidación, vallado y señalización de los restos arqueológicos, se requiere una intensa labor de difusión para ayudar a los visitantes a adentrarse en una época o tiempo pasados, lo cual implica la reconstrucción -física o virtual- de determinados edificios e infraestructuras, a través de planos, maquetas, vídeos, exposiciones o paneles informativos. Así pues, como indica A. Pérez-Juez (1999), "entre la conservación de un yacimiento y el establecimiento de un itinerario didáctico existe un amplio repertorio que posibilita que los yacimientos arqueológicos se conviertan en centros pedagógicos de primera mano para la enseñanza de la Historia de forma amena e interesante, en donde formación y ocio no son incompatibles". De igual forma, los museos arqueológicos deberían ofrecer, junto a los talleres centrados en la manipulación de objetos, acciones que potencien aún más el desarrollo de las habilidades mentales de los individuos, como la imaginación y la capacidad de deducción.

Es en estos espacios, además, donde se materializa a la perfección la necesidad de tener a las Nuevas Tecnologías de fieles aliadas, por estar más vinculadas a las nuevas generaciones y a sus métodos de aprendizaje $\mathrm{e}^{13}$, y por poder ofrecer mayor objetividad y riqueza de matices en entornos interactivos de gran atractivo ${ }^{14}$. Por otra parte, la introducción de las prácticas propias de la Arqueología Experimental en las escuelas puede también favorecer la identificación y el respeto por el Patrimonio, así como la mejor comprensión de los vestigios arqueológicos, a menudo relegados a piezas descontextualizadas en las vitrinas de los museos (BARDAVIO 1998; MARTIN et alii

13 En estas iniciativas es imprescindible contemplar no sólo los conceptos didácticos, sino aquéllos de carácter científico que se encuentran en la base de la disciplina arqueológica; de lo contrario, la divulgación perderá todo su valor y se convierte en un puro entretenimiento (VELÁZQUEZ, CONDE, BAENA 2004).

14 Siguiendo esta premisa hemos creado el videojuego Arqueología Virtual. Aprende a ser arqueólogo y realizado en varias ocasiones -con ayuda de tabletas y herramientas digitales- nuestra Gymkana Virtual por el Casco Histórico de Córdoba. 
2012). Como consecuencia natural de la Arqueología Experimental encontramos el Living Archaeology o Living History, traducido como "reconstrucción histórica" o "historia viva"; un modo de difundir el pasado nacido en tierras británicas y difundido rápidamente por todo el mundo, aplicándose hoy en día a todos los períodos históricos, desde la Prehistoria hasta la Segunda Guerra Mundial. A diferencia de otras propuestas populares repletas de anacronismos, en las que grupos de actores recitan textos, se "disfrazan" o participan en "mercadillos" romanos o medievales organizados sin ningún criterio, las recreaciones históricas nos permiten interactuar y revivir literalmente escenas cotidianas y momentos de nuestra Historia fundamentados en sólidas bases científicas. Por último, el apadrinamiento de monumentos, derivado del proyecto internacional La scuola adotta nacido en Nápoles en 1992, se perfila como uno de los caminos más provechosos para desarrollar actitudes de defensa y valorización de los bienes patrimoniales entre los más jóvenes ${ }^{15}$. Para ello se precisa el establecimiento de sinergias entre los principales organismos, públicos o privados, de los que depende el Patrimonio, especialmente administraciones, escuelas e instituciones académicas, pero sin olvidar la implicación activa de la sociedad.

En definitiva, en la medida en que la Arqueología -en el sentido más amplio de la palabra- y la Escuela se aproximen y participen conjuntamente en dinámicas divulgativas y educativas, nuestro Patrimonio Histórico-Arqueológico saldrá beneficiado (FERNÁNDEZ OCHOA et alii 1990), aun con el riesgo añadido de incrementar la afición por el coleccionismo por parte de los profanos o de fracasar estrepitosamente en nuestras intenciones, por cuanto resulta imposible educar al que no quiere ser educado. Si bien, como indica M. Luque Cortina (en prensa) al referirse a los objetivos de futuro planteados para Arqueopinto: "en 2037 un rango de escolares que comienzan este año la educación infantil tendrán entonces entre 25 y 30 años y serán más de 3 millones de personas, una buena parte de los futuros visitantes de museos, yacimientos y monumentos relacionados con el patrimonio arqueológico en los que las administraciones invierten actualmente grandes cantidades de dinero. Si nos preguntamos cuál será en el futuro su actitud hacia ese legado que construimos hoy probablemente no tengamos clara la respuesta, y sin embargo deberíamos, ya que les estamos educando para ello".

\section{Bibliografía}

A. BARDAVIO NOVI: "Arqueología Experimental en la ESO". Revista de Arqueología, 208 (1998), 6-15.

15 Para más información, ténganse en cuenta las experiencias de Patrimonitos o Jóvenes Embajadores por el Patrimonio. 
A. BARDAVIO NOVI; P. GONZÁLEZ MARCÉN: "La Arqueología y la Prehistoria como eje en un proyecto integrado de comprensión del territorio. El ejemplo del Campo de Aprendizaje de La Noguera". R. M. ÁVILA RUIZ.; M. A. CRUZ RODRÍGUEZ; M. C. DÍEZ BEDMAR (Eds.): Didáctica de las Ciencias Sociales, Currículo Escolar y Formación del Profesorado. La didáctica de las Ciencias Sociales en los nuevos planes de estudio. Universidad de Jaén, Jaén: 2008, 473-484.

F. CRIADO BOADO; M. M. BÓVEDA LÓPEZ: "La formación de postgrado en Arqueología y gestión del Patrimonio. La experiencia del Laboratorio de Arqueología y Formas Culturales". Treballs d'Arqueologia, 6 (2000), pp. 176204.

J. DAVID: "L'Archeodrome de Bourgogne: Vings ans aprés...". Treballs d'Arqueologia, 5 (1998), 115-123.

C. FERNÁNDEZ OCHOA; M. V. GALLEGO GUITIAN; M. DOMÍNGUEZ SUÁREZ; A. ROMERO MASÍA (1990): Arqueología. Enseñar desde las raíces de la Historia, Narcea Ediciones, Instituto de Estudios Pedagógicos Somosaguas, Madrid: 1990.

J. GARCÍA ROSSELLÓ; D. JAVALOYAS MOLINA; D. ALBERO SANTACREU; M. CALVO TRÍAS: "El aprendizaje en grupo fuera del aula: los talleres didácticos de Arqueología". UNIVEST-2011, Gerona, recurso on-line, (2011).

A. GIL; M. I. IZQUIERDO; C. PÉREZ; S. FIÉRREZ: "La simulación arqueológica como instrumento didáctico: la experiencia del taller de arqueología 4 de Valencia". Treballs d'Arqueologia, 4 (1996), 143-161.

M. GRIPENBERG; E. LIZARTE: "El sistema educativo en Finlandia y su éxito en la prueba PISA". Journal for Educators, Teachers and Trainers, 3 (2012), 14-24.

F. LÓPEZ AMBITE: "La arqueología en la educación secundaria". Innovación Educativa, 10 (2000), pp. 343-35.

M. LUQUE CORTINA: "ARQUEOPINTO. Dinamización del patrimonio arqueológico. Claves para la gestión sostenible desde la perspectiva empresarial". VII Jornadas de Patrimonio Arqueológico de la Comunidad de Madrid, noviembre - 2011: en prensa.

B. MADSEN: "An experimental site in Lejre". Flintknappers' Exchange, 4-1 (1981), 16-20.

D. MARTíN PUIG; F. CUARTERO MONTEAGUDO: "Los talleres didácticos de Prehistoria y Arqueología Experimental del Museo de los Orígenes (Casa de San Isidro)". Apuntes de Arqueología, 24 (2008), 12-18.

D. MARTÍN PUIG; F. CUARTERO MONTEAGUDO; D. RUBIO GIL; D. SANMARTÍ ESTEBAN: "De la investigación a la difusión: la experimentación como recurso didáctico en la elaboración de modelos de divulgación y puesta en valor del 
Ruiz Osuna, A. y Vázquez Navajas, A. - El aula de formación permanente...

patrimonio arqueológico". Boletín de Arqueología Experimental, 9 (2012), 125-131.

J. A. MARTíN RUIZ: "Simulación arqueológica en escuelas taller y casas de oficio". Revista de Arqueología, 234 (2000), 6-11.

P. MINARD; F. FOLCHER: Guédelon: Des hommes fous, un chateau fort. Paris: 2003.

J. MORER; M. C. BELARTE; J. SANMARTÍ; J. SANTACANA: "El laboratori d'arqueologia experimental del Vendrell (Baix Penedès). Primers resultats". Pyrenae, 30 (1999), 123-145.

M. J. NOAIN: "El taller de verano «Arqueología y Arquitectura», Monte Urgull, Donostia-San Sebastián". Boletín de Arqueología Experimental, 5 (2002-2003), 10-14.

C. R. OLARIA I PUYOLES: “La prehistoria de la cerámica". Millars: Espai i historia, 17 (1994), 171-184

A. PÉREZ-JUEZ GIL: "El yacimiento como recurso didáctico: acondicionamiento, restauración, reconstrucción y otras ideas". Apuntes de Arqueología del Boletín del Ilustre Colegio Oficial de Doctores y Licenciados de Madrid, 7 (1999), 1922.

J. POU; J. SANMARTÍ; J. SANTACANA: "La reconstrucció del poblat ibèric d'Alorda Park o de les Toixoneres (Calafell, Baix Penedès)", Tribuna d'Arqueologia (1993-1994), 51-62.

P. REYNOLDS: Iron-age farm: the Butser experiment. British Museum Publications: 1979.

P. REYNOLDS: "L'experiment en arqueología i I'antiga granja Butser". Tribuna d'Arqueologia (1988-89), 131-138.

S. ROVIRA: "Arqueometalurgia experimental en el departamento de Prehistoria y Arqueología de la U.A.M.". Cuadernos de Prehistoria y Arqueología de la Universidad Autónoma de Madrid, 37-38 (2011-12), 105-120.

G. RUIZ ZAPATERO: "El pasado excluido: la enseñanza de la Historia nates de la aparición de la escritura". Iber: Didáctica de las ciencias sociales, geografía e historia, 6 (1995), 19-30.

R. VELÁZQUEZ RAYÓN; C. CONDE RUIZ; J. BAENA PREYSLER: "La Arqueología Experimental en el Museo de San Isidro: Talleres didácticos para escolares". Estudios de Prehistoria y Arqueología Madrileñas, 13 (2004), 3-17. 